hep-th/0412183

KCL-MTH-04/16

ITP-UU-04/51

SPIN-04/33

UG-04/04

\title{
Non-extremal instantons and wormholes in string theory
}

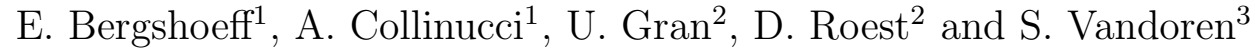 \\ ${ }^{1}$ Centre for Theoretical Physics, University of Groningen, Nijenborgh 4, 9747 AG Groningen, \\ The Netherlands \\ e.a.bergshoeff, a.collinucci@phys.rug.nl \\ ${ }^{2}$ Department of Mathematics, King's College London, Strand, London, WC2R 2LS, United \\ Kingdom \\ ugran, droest@mth.kcl.ac.uk \\ ${ }^{3}$ Institute for Theoretical Physics and Spinoza Institute \\ Utrecht University, 3508 TD Utrecht, The Netherlands \\ s.vandoren@phys.uu.nl
}

\begin{abstract}
We construct the most general non-extremal spherically symmetric instanton solution of a gravity-dilaton-axion system with $S L(2, R)$ symmetry, for arbitrary euclidean spacetime dimension $D \geq 3$. A subclass of these solutions describe completely regular wormhole geometries, whose size is determined by an invariant combination of the $S L(2, R)$ charges. Our results can be applied to four-dimensional effective actions of type II strings compactified on a Calabi-Yau manifold, and in particular to the universal hypermultiplet coupled to gravity. We show that these models contain regular wormhole solutions, supported by regular dilaton and RR scalar fields of the universal hypermultiplet.
\end{abstract}

\section{The action}

This paper contains a summary of [1]. In addition, we demonstrate the existence of regular wormhole solutions in the universal hypermultiplet, which is present in the low-energy effective action of type II string theories compactified on a Calabi-Yau manifold. Wormhole 
solutions from Calabi-Yau compactifications were also found in [2]. The main difference with our solution is that in our case we also include RR fields, and furthermore, our solution is regular in the complete domain of the wormhole geometry.

We start with the action of a gravity-dilaton-axion system in $D$-spacetime dimensions. In Minkowski space, the Lagrangian is

$$
\mathcal{L}_{M}=\frac{1}{2} \sqrt{|g|}\left[R-\frac{1}{2}(\partial \phi)^{2}-\frac{1}{2} e^{b \phi}(\partial \chi)^{2}\right]
$$

where $b$ is an arbitrary dilaton coupling parameter. This Lagrangian has an $S L(2, R)$ group of symmetries. They can be realized as modular transformations on the complex field

$$
\tau \equiv \frac{b}{2} \chi+i e^{-b \phi / 2}, \quad \tau \rightarrow \frac{\alpha \tau+\beta}{\gamma \tau+\delta}, \quad \alpha \delta-\beta \gamma=1
$$

and is valid for any nonzero value of the dilaton coupling $b$.

This theory occurs for example as the scalar section of IIB supergravity in $D=10$ Minkowski space-time with dilaton-coupling parameter $b=2$. Other values of $b$ can arise when considering (truncations of) compactifications of II supergravity. The main example we will discuss here is that of the universal hypermultiplet, that arises after compactifying type IIA strings on a (rigid) Calabi-Yau threefold down to $D=4$. This hypermultiplet contains four scalars, $\phi$ and $\sigma$ coming from the NS sector, and $\psi$ and $\varphi$ coming from the $\mathrm{RR}$ sector. The four-dimensional Lagrangian can be written as

$$
\mathcal{L}_{M}=\frac{1}{2} \sqrt{|g|}\left[R-\frac{1}{2}(\partial \phi)^{2}-\frac{1}{2} e^{\phi}\left((\partial \psi)^{2}+(\partial \varphi)^{2}\right)-\frac{1}{2} e^{2 \phi}(\partial \sigma+\psi \partial \varphi)^{2}\right]
$$

The scalar symmetry group is now $S U(2,1)$, but contains various inequivalent $S L(2, R)$ subgroups. For instance, if we set both $\sigma=\psi=0$, we get (1.1) whith $b=1$ whereby $\varphi$ is identified with $\chi$. If we set $\psi=\varphi=0$, we have $b=2$ and $\sigma$ is identified with $\chi$. By writing down the field equations for (1.3), it is easy to see that these truncations are consistent. Extremal instantons in the universal hypermultiplet have been discussed in detail in [3, 4, 5], and correspond to wrapped (euclidean) membranes along three-cycles, or wrapped NS5-branes along the entire Calabi-Yau. These two cases correspond to $b=1$ and $b=2$ respectively. Using the results obtained in [1], we will here generalize this to the non-extremal case, and show that there are interesting and new solutions that have the spacetime geometry of a wormhole.

To discuss instantons, we first have to perform a Wick rotation. This rotation is best understood by dualizing the axion into a $(D-2)$-form potential. One then finds that under a Wick rotation, $\chi \rightarrow i \chi$. The Euclidean Lagrangian corresponding to (1.1) is then

$$
\mathcal{L}_{E}=\frac{1}{2} \sqrt{g}\left[R-\frac{1}{2}(\partial \phi)^{2}+\frac{1}{2} e^{b \phi}(\partial \chi)^{2}\right]
$$


with all fields real. Notice that in the scalar formulation, as opposed to the formulation with the $(D-1)$-form field strength, the contribution to the action coming from the scalar sector is not positive definite. For $b=2$ and $D=10$ this is the gravity-scalar part of Euclidean IIB supergravity, in which the D-instanton can easily be found as a solution of the Euclidean equations of motion [6, 7]. The non-extremal solutions were found in [1], and we repeat them in the next section.

As already explained, compactifications of string theory can give rise to other values of b. The Euclidean version of the universal hypermultiplet Lagrangian (1.3) can best be understood in terms of the double-tensor multiplet formulation, in which $\varphi$ and $\sigma$ are dualized into two antisymmetric tensors [3]. After a Wick rotation, $\varphi \rightarrow i \varphi, \sigma \rightarrow i \sigma$, and the Euclidean Lagrangian for the universal hypermultiplet becomes

$$
\mathcal{L}_{E}=\frac{1}{2} \sqrt{|g|}\left[R-\frac{1}{2}(\partial \phi)^{2}-\frac{1}{2} e^{\phi}\left((\partial \psi)^{2}-(\partial \varphi)^{2}\right)+\frac{1}{2} e^{2 \phi}(\partial \sigma+\psi \partial \varphi)^{2}\right]
$$

Notice that the two truncations, $\psi=\sigma=0$ and $\psi=\varphi=0$, both fall into the class of (1.4), in which we have $b=1$ and $b=2$ respectively.

There are three conserved currents for the $S L(2, R)$ transformations in the Euclidean model, satisfying $\nabla_{\mu} j^{\mu}=0$. The corresponding charges are denoted by $q_{3}, q_{+}$and $q_{-}$, and are normalized as specified in [1]. They transform under $S L(2, R)$ in such a way that the combination

$$
\mathrm{q}^{2} \equiv q_{3}^{2}-q_{+} q_{-},
$$

is invariant [8, 1]. The three conjugacy classes of $S L(2, R)$ then correspond to $\mathrm{q}^{2}<0, \mathrm{q}^{2}=0$ and $\mathrm{q}^{2}>0$. The extremal solutions will have $\mathrm{q}^{2}=0$, the non-extremal $\mathrm{q}^{2} \neq 0$. The wormhole solutions will have $\mathrm{q}^{2}<0$. For later convenience, it is useful to define the quantity

$$
c \equiv \sqrt{\frac{2(D-1)}{D-2}}
$$

which will appear explicitly in the instanton solutions below.

\section{Instanton solutions}

We search for generalised instanton solutions with manifest $S O(D)$ symmetry,

$$
d s^{2}=e^{2 B(r)}\left(d r^{2}+r^{2} d \Omega_{D-1}^{2}\right), \quad \phi=\phi(r), \quad \chi=\chi(r) .
$$

The standard D-instanton solution [6] is obtained for the special case that $B(r)$ is constant. Other references on generalised instantons and wormholes that are related to our work are [2, 9, 10, 11, 12, 13, 14, 15, 16, 17]. To obtain an $S O(D)$ symmetric generalised instanton 
solution, we allow for a non-constant $B(r)$ and solve the field equations following from the Euclidean action (1.4). This was done in detail in [1]. Here we summarise the result.

The solution can be written in a compact form by using a harmonic function $H(r)$ over a conformally flat space with metric as given in (2.1),

$$
H(r)=\frac{b c}{2} \log \left(f_{+}(r) / f_{-}(r)\right), \quad B(r)=\frac{1}{D-2} \log \left(f_{+} f_{-}\right), \quad f_{ \pm}(r)=1 \pm \frac{\mathrm{q}}{r^{D-2}},
$$

The general instanton solution can then be written as

$$
\begin{aligned}
d s^{2} & =\left(1-\frac{\mathrm{q}^{2}}{r^{2(D-2)}}\right)^{2 /(D-2)}\left(d r^{2}+r^{2} d \Omega_{D-1}^{2}\right) \\
e^{b \phi(r)} & =\left(\frac{q_{-}}{\mathrm{q}} \sinh \left(H(r)+C_{1}\right)\right)^{2} \\
\chi(r) & =\frac{2}{b q_{-}}\left(\mathrm{q} \operatorname{coth}\left(H(r)+C_{1}\right)-q_{3}\right) .
\end{aligned}
$$

This solution is valid for any value ${ }^{1}$ of $b \neq 0$. The integration constant $C_{1}$ can be traded for the asymptotic value of the dilaton that we will later identify with the string coupling constant. Notice also the explicit dependence on the $S l(2, R)$ charges $q_{3}, q_{-}$and $q_{+}$. The solutions (2.3) are valid both for $\mathrm{q}^{2} \equiv q_{3}^{2}-q_{-} q_{+}$positive, negative and zero, corresponding to the three conjugacy classes of $S L(2, R)$. We now discuss these three cases separately:

\section{- $\mathrm{q}^{2}>0$ : Black Holes}

In this case $\mathrm{q}$ is real and the solution is given by (2.3) with all constants real. However, the metric becomes imaginary below a critical radius

$$
r^{D-2}<r_{c}^{D-2}=\mathbf{q}
$$

One can check that there is a curvature singularity at $r=r_{c}$, which happens at strong string coupling: $e^{\phi(r)} \rightarrow \infty$ as $r \rightarrow r_{c}$.

Between $r=r_{c}$ and $r=\infty, H$ varies between $\infty$ and 0 , and with an appropriate choice of $C_{1}$, i.e. a positive value of $C_{1}$, the scalars have no further singularities in this domain. Thus one might hope to have a modification of this solution by higherorder contributions to the effective action of IIB string theory [13]. Alternatively, one can consider the possible resolution of this singularity upon uplifting to one higher dimension. In [1], we showed that this indeed happens for the special case of

$$
b \geq \sqrt{\frac{2(D-2)}{D-1}}
$$

\footnotetext{
${ }^{1}$ The case $b=0$ is treated in [18].
} 
equivalent to $b c \geq 2$. Upon uplifting, this becomes a non-extremal dilatonic black hole. The case when $b c=2$ lifts up to a (non-dilatonic) Reissner-Nordström black hole with mass and charge given by

$$
Q=-2 q_{-}, \quad M=2 \sqrt{\mathrm{q}^{2}+q_{-}^{2}} \quad \Rightarrow \quad \mathrm{q}^{2}=\frac{M^{2}-Q^{2}}{4} .
$$

Hence, the $\mathrm{q}^{2}>0$ solutions with $b c \geq 2$ are spatial sections of a higher-dimensional (Lorentzian) black hole solution. The case of $b c<2$ cannot be uplifted and remain singular instanton solutions in $D$-dimensions. In Einstein frame, these geometries are singular wormholes that are pinched at the selfdual radius $r_{\mathrm{sd}}=r_{c}$ [1].

In the case of $q^{2}>0$, there is an interesting limit in which $q_{-} \rightarrow 0$. This yields a solution with only two independent integration constants, $q_{+}$and $q^{2}$. The range of validity of this solution is equal to that of the above solution with $q_{-} \neq 0$ : it is well-defined for $r>r_{c}$, while at $r=r_{c}$ the metric has a singularity and the dilaton blows up. The singularity can be resolved upon uplifting for all values of $b c \geq 2$ to Schwarzschild black holes, with mass $M=2 \mathrm{q}$. More details can be found in [1].

- $\mathrm{q}^{2}=0$ : Extremal instantons

We now consider the limit $\mathrm{q}^{2} \rightarrow 0$ of the general solution (2.3), after rescaling the constant $C_{1}$ with a factor $\mathrm{q}$ to make the limit well-defined. Taking the limit yields the extremal solution:

$$
d s^{2}=d r^{2}+r^{2} d \Omega_{D-1}^{2}, \quad e^{b \phi(r) / 2}=h \quad \chi(r)=\frac{2}{b}\left(h^{-1}-\frac{q_{3}}{q_{-}}\right),
$$

where $h(r)$ is the harmonic function:

$$
h(r)=g_{s}^{b / 2}+\frac{b c q_{-}}{r^{D-2}},
$$

and $g_{s}$ is the asymptotic value of the dilaton at infinity.

This is the extremal D-instanton solution of [6]. This solution is regular over the

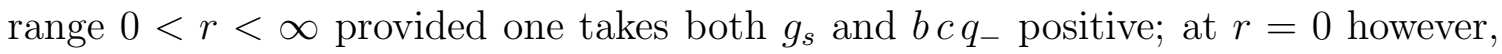
the harmonic function blows up and the scalars are singular. Similar to the case of $\mathrm{q}^{\mathbf{2}}>\mathbf{0}$, these singular solutions can be lifted to higher dimensions where, e.g. for $b c=2$, they become extremal Reissner-Nordström black holes.

- $\mathrm{q}^{2}<0$ : Wormholes

In this case $\mathrm{q}$ is imaginary. To obtain a real solution we must take $C_{1}$ to be imaginary. We therefore redefine

$$
\mathrm{q} \rightarrow i \tilde{\mathrm{q}} \quad C_{1} \rightarrow i \tilde{C}_{1}
$$


such that $\tilde{\mathrm{q}}$ and $\tilde{C}_{1}$ are real. One can now rewrite the solution (2.3) by using the relation

$$
\log \left(f_{+} / f_{-}\right)=2 \operatorname{arctanh}\left(\mathbf{q} / r^{D-2}\right),
$$

and, next, replacing the hyperbolic trigonometric functions by trigonometric ones in such a way that no imaginary quantities appear. We thus find that, for $\mathrm{q}^{2}<0$, the general solution (2.3) takes the following form:

$$
\begin{aligned}
d s^{2} & =\left(1+\frac{\tilde{\mathrm{q}}^{2}}{r^{2(D-2)}}\right)^{2 /(D-2)}\left(d r^{2}+r^{2} d \Omega_{D-1}^{2}\right), \\
e^{b \phi(r)} & =\left(\frac{q_{-}}{\tilde{\mathrm{q}}} \sin \left(b c \arctan \left(\frac{\tilde{\mathrm{q}}}{r^{D-2}}\right)+\tilde{C}_{1}\right)\right)^{2}, \\
\chi(r) & =\frac{2}{b q_{-}}\left(\tilde{\mathrm{q}} \cot \left(b c \arctan \left(\frac{\tilde{\mathrm{q}}}{r^{D-2}}\right)+\tilde{C}_{1}\right)-q_{3}\right) .
\end{aligned}
$$

The metric and curvature are well behaved over the range $0<r<\infty$. However, the scalars can only be non-singular over the same range by an appropriate choice of $\tilde{C}_{1}$ provided that $b c<2$. This can be seen as follows. The arctan varies over a range of $\pi / 2$ when $r$ goes from 0 to $\infty$. It is multiplied by $b c$ and thus the argument of the sine varies over a range of more than $\pi$ if $b c>2$. Therefore, for $b c>2$ there is always a point $r_{c}$ such that $\chi \rightarrow \infty$ as $r \rightarrow r_{c}$. Note that the breakdown of the solution occurs at weak string coupling: $e^{\phi} \rightarrow 0$ as $r \rightarrow r_{c}$. This singularity is not resolved upon uplifting and corresponds to a black hole with a naked singularity (in the case of Reissner-Nordström, $M^{2}<Q^{2}$. The same holds for the liming case of $b c=2$. Therefore the case $\mathrm{q}^{2}<0$ only yields regular instanton solutions for $b c<2$, together with the condition that $\tilde{C}_{1}$ and $\tilde{C}_{1}+b c \pi / 2$ are on the same branch of the cotangent.

The metric in (2.11) has a $Z_{2}$ isometry corresponding to the reflection $r^{D-2} \rightarrow \tilde{\mathbf{q}} r^{2-D}$ which interchanges the two asymptotically flat regions. This reflection has a fixed point, corresponding to the selfdual radius

$$
r_{\mathrm{sd}}^{D-2}=\tilde{\mathrm{q}}
$$

Furthermore, the thickness of the neck was in [1] computed to be

$$
\rho_{\mathrm{sd}}^{D-2}=2 \tilde{\mathrm{q}} .
$$

We have summarised this in the following figure: 


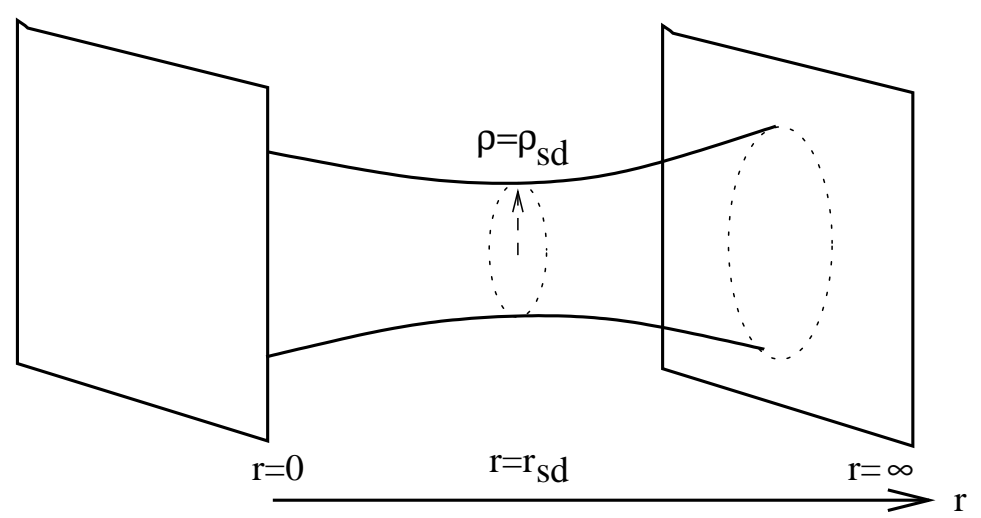

Figure 1: The geometry of a wormhole. The two asymptotically flat regions at $r=0$ and $r=\infty$ are connected via a neck with a minimal physical radius $\rho_{\text {sd }}$ at the self-dual radius $r_{s d}$.

\section{Instanton action}

The value of the action, evaluated on the instanton solution, is a key ingredient in the semiclassical approximation of the euclidean path integral. In [1] we computed the instanton action for the three cases, corresponding to $\mathrm{q}^{2}>0, \mathrm{q}^{2}=0$, and $\mathrm{q}^{2}<0$. This was done by specifying the additional surface term added to (1.4), which solely determines the instanton action. This surface term can be found from the dual description in terms of the $(D-1)$-form field strength formulation. We here summarise the results.

For the case when $q^{2} \geq 0$, the contribution to the action coming from infinity is given by

$$
\mathcal{S}_{\text {inst }}^{\infty}=\frac{4}{b^{2}}(D-2) \mathcal{V} \operatorname{ol}\left(S^{D-1}\right) b c\left(\sqrt{\mathrm{q}^{2}+\frac{q_{-}^{2}}{g_{s}^{b}}}\right) .
$$

Here we have used the relation between $C_{1}$ and the asymptotic value of the dilaton, $g_{s}^{b}=$ $\left(q_{-} / \mathrm{q}\right)^{2} \sinh ^{2} C_{1}$. Notice that the instanton action is proportional to the mass of the black hole to which the solution uplifts in one dimension higher. Furthermore, the result (3.1) also hols for $\mathrm{q}^{2}=0$, which gives the lowest value of the action. The resulting instanton action is then inversely proportional to $g_{s}^{b / 2}$. The D-instanton of ten-dimensional IIB corresponds to taking $b=2$. The extremal instantons for the universal hypermultiplet action (1.3) [3, 4] also fall into this class: the membrane instantons correspond to $b=1$ whereas the NS-fivebrane instantons correspond ${ }^{2}$ to $b=2$. We have here given only the contribution from infinitiy. The non-extremal instantons also contribute to the action at

\footnotetext{
${ }^{2}$ To compare with 3, 4, one has to redefine the string coupling constant by taking the square root. We correct here a minor mistake in [1], in which the membrane and fivebrane instantons were written to correspond to $b=2$ and $b=4$.
} 
the other boundary, where $r=r_{c}$. Since the solution is singular at this point, it is however not clear that the supergravity approximation is still valid in this region.

The case when $\mathrm{q}^{2}<0$ is very different. For $b c<2$, these are regular wormhole solutions with two asymptotic boundaries at $r=0$ and $r=\infty$ that are related by a reflection symmetry. The wormhole action gets contributions from both these boundaries, and the result is

$$
\mathcal{S}_{\text {wormhole }}=\frac{4}{b^{2}}(D-2) \mathcal{V} \text { ol }\left(S^{D-1}\right) b c \tilde{\mathrm{q}}\left(\cot \tilde{C}_{1}-\cot \left(\tilde{C}_{1}+b c \frac{\pi}{2}\right)\right)
$$

Due to the fact that $\tilde{C}_{1}$ and $\tilde{C}_{1}+b c \pi / 2$ are on the same branch of the cotangent, the total instanton action is manifestly positive definite. One can rewrite the above result in terms of the string coupling constant, using $g_{s}^{b / 2} \equiv e^{b \phi_{\infty} / 2}=\left(q_{-} / \tilde{\mathrm{q}}\right) \sin \tilde{C}_{1}$. In the neighborhood of $b c \approx 2$, the instanton action becomes very large, and in the limit to the critical point where $b c=2$, it diverges. At that point, the wormhole solution is no longer regular.

\section{Wormholes in string theory}

We have seen that the condition for regular wormholes is that there exist models for which $b c<2$. In type IIB in ten dimensions, this is not satisfied. Toroidal compactifications of string theory only lead to values of $b$ for which $b c \geq 2$, so no wormholes exist for these cases. However, we have seen that for the universal hypermultplet, which descends from a Calabi-Yau compactification of type II strings, one can have the value $b=1$ in $D=4$, and so $b c=\sqrt{3}<2$. The solution is then characterized by the dilaton and the RR scalar $\varphi$ that descends from the RR three-form gauge potential in IIA in ten dimensions. Since the extremal case $\mathrm{q}^{2}=0$ corresponds to a wrapped type IIA euclidean membrane over a (supersymmetric) three-cycle, it is natural to suggest that the wormhole, with $\mathrm{q}^{2}<0$, corresponds to a wrapped non-extremal euclidean D2 brane.

\section{Acknowledgement}

It is a pleasure to thank Mathijs de Vroome for stimulating discussions.

This work is supported in part by the Spanish grant BFM2003-01090 and the European Community's Human Potential Programme under contract HPRN-CT-2000-00131 Quantum Spacetime, in which E.B. and D.R. are associated to Utrecht University. The work of U.G. is funded by the Swedish Research Council. 


\section{References}

[1] E. Bergshoeff, A. Collinucci, U. Gran, D. Roest and S. Vandoren, Non-extremal Dinstantons, JHEP 0410:031, 2004, hep-th/0406038.

[2] S. B. Giddings and A. Strominger, String Wormholes, Phys. Lett. B230 (1989) 46.

[3] U. Theis and S. Vandoren, Instantons in the double-tensor multiplet, JHEP 09 (2002) 059, hep-th/0208145.

[4] M. Davidse, M. de Vroome, U. Theis and S. Vandoren, Instanton solutions for the universal hypermultiplet, Fortsch. Phys. 52 (2004) 696-701, hep-th/0309220.

[5] M. Davidse, U. Theis and S. Vandoren, Fivebrane instanton corrections to the universal hypermultiplet, Nucl. Phys. B697 (2004), 48, hep-th/0404147.

[6] G. W. Gibbons, M. B. Green and M. J. Perry, Instantons and Seven-Branes in Type IIB Superstring Theory, Phys. Lett. B370 (1996) 37-44, hep-th/9511080.

[7] M. B. Green and M. Gutperle, Effects of D-instantons, Nucl. Phys. B498 (1997) 195-227, hep-th/9701093.

[8] E. Bergshoeff, U. Gran and D. Roest, Type IIB seven-brane solutions from nine-dimensional domain walls, Class. Quant. Grav. 19 (2002) 4207-4226, hep-th/0203202.

[9] S. J. Rey, The Confining Phase Of Superstrings And Axionic Strings, Phys. Rev. D 43 (1991) 526.

[10] D. H. Coule and K. i. Maeda, Class. Quant. Grav. 7 (1990) 955.

[11] J. Y. Kim, H. W. Lee and Y. S. Myung, D-instanton and D-wormhole, Phys. Lett. B400 (1997) 32-36, hep-th/9612249.

[12] E. Bergshoeff and K. Behrndt, D-instantons and asymptotic geometries, Class. Quant. Grav. 15 (1998) 1801-1813, hep-th/9803090.

[13] M. B. Einhorn, Instantons of type IIB supergravity in ten dimensions, Phys. Rev. D66 (2002) 105026, hep-th/0201244.

[14] M. Gutperle and W. Sabra, Instantons and wormholes in Minkowski and (A)dS spaces, Nucl. Phys. B647 (2002) 344-356, hep-th/0206153. 
[15] M. B. Einhorn, Instantons and SL(2,R) symmetry in type IIB supergravity, Phys. Rev. D68 (2003) 067701, hep-th/0212322.

[16] J. Y. Kim, Y. b. Kim and J. E. Hetrick, Classical stability of stringy wormholes in flat and AdS spaces, hep-th/0301191.

[17] J. Maldacena and L. Maoz, Wormholes in AdS, JHEP 0402, 053 (2004), hep-th/0401024.

[18] R. C. Myers, New axionic instantons in quantum gravity, Phys. Rev. D38 (1988) 1327; Axionic wormholes, In *Perth 1988, Proceedings, Recent developments in theoretical and experimental general relativity, gravitation and relativistic field theories, pt. A* 441-444. 